\title{
l-SIMPLE LATTICE-ORDERED GROUPS
}

\author{
by A. M. W. GLASS $\dagger$ \\ (Received 9th May 1973)
}

\section{Introduction}

Let $G$ be a lattice-ordered group ( $l$-group) and $H$ a subgroup of $G . \quad H$ is said to be an l-subgroup of $G$ if it is a sublattice of $G . H$ is said to be convex if $h_{1}, h_{2} \in H$ and $h_{1} \leqq g \leqq h_{2}$ imply $g \in H$. The normal convex $l$-subgroups ( $l$-ideals) of an $l$-group play the same role in the study of lattice-ordered groups as do normal subgroups in the investigation of groups. For this reason, an $l$-group is said to be $l$-simple if it has no non-trivial $l$-ideals. As in group theory, a central task in the examination of lattice-ordered groups is to characterise those $l$-groups which are $l$-simple.

Let $\langle S, \leqq\rangle$ be a totally ordered set. Aut $(\langle S, \leqq\rangle)$ is an l-group under the ordering: $f \leqq g$ if and only if $f(s) \leqq g(s)$ for all $s \in S$. Also

$$
(g \vee h)(s)=\max \{g(s), h(s)\} \text { and }(g \wedge h)(s)=\min \{g(s), h(s)\} \text {. }
$$

Theorem (Holland (3) Theorem 2). If $G$ is an l-group, there exists a totally ordered set $\langle S, \leqq\rangle$ such that $G$ can be l-embedded (a group embedding which preserves the lattice operation) in Aut $(\langle S, \leqq\rangle)$.

This theorem is the analogue of the theorem of Cayley for groups and, in this case, we will say that $G$ is represented on $\langle S, \leqq\rangle$.

For the rest of this paper $\langle S, \leqq\rangle$ will denote a totally ordered set and Aut $(\langle S$, $\\rangle)$ will be the $l$-group described above.

Let $G$ be an $l$-subgroup of Aut $(\langle S, \leqq\rangle) . \quad G$ is said to be transitive on $S$ if, for any $s, t \in S$, there exists $g \in G$ such that $g(s)=t$.

Theorem ((3), Corollary 2). Every l-simple l-group can be represented transitively on some set $\langle S, \leqq\rangle$.

An equivalence relation $\mathscr{E}$ on $\langle S, \leqq\rangle$ is called a convex $G$-congruence on $\langle S, \leqq\rangle$ if each equivalence class of $\mathscr{E}$ is convex and, for all $s, t \in S, g(s) \mathscr{E} g(t)$ for all $g \in G$ whenever $s \mathscr{E} t$. If there are no non-trivial convex $G$-congruences on $\langle S, \leqq\rangle$, then $G$ is said to be $o$-primitive on $\langle S, \leqq\rangle$.

In attempting to classify the $l$-simple $l$-groups, the first step is to characterise those that have a transitive $o$-primitive representation.

Let $G$ be an $l$-subgroup of Aut $(\langle S, \leqq\rangle) . \quad G$ is $o$-2 transitive on $\langle S, \leqq\rangle$ if,

† This research was supported by a grant from the Bowling Green State University Faculty Research Committee. The author is also grateful to W. Charles Holland for some helpful discussions. 
for all $s, t, u, v \in S$ such that $s<t$ and $u<v$, there exists $g \in G$ such that $g(s)=u$ and $g(t)=v$. Let $\langle\bar{S}, \leqq\rangle$ be the Dedekind closure of $\langle S, \leqq\rangle$. If there exists a positive $f_{0} \in$ Aut $(\langle\bar{S}, \leqq\rangle)$ such that $f_{0}$ generates the centraliser of $G$ in Aut $\left(\langle\bar{S}, \leqq\rangle\right.$ ) and $\left\{f_{0}^{n}\left(s_{0}\right): n \in \mathbf{Z}\right\}$ is unbounded (above and below) in $\langle\bar{S}, \leqq\rangle$ for some $s_{0} \in S$, then $G$ is said to be periodic and $f_{0}$ is called the period of $G$.

Theorem (Holland (4) and McCleary (7)). Let $G$ be a transitive o-primitive l-subgroup of Aut $(\langle S, \leqq\rangle)$. Then either:

(i) $G$ is a subgroup of the real numbers $(\langle\mathbf{R}, \leqq\rangle)$ in its regular representation;

or (ii) $G$ is periodic;

or (iii) $G$ is $o-2$ transitive on $\langle S, \leqq\rangle$.

If $G$ falls into either of the first two categories, it is $l$-simple (see (7)). Let $g \in G$. The support of $g$ is the set of points of $S$ moved by $g$; i.e.

$$
\operatorname{supp}(g)=\{s \in S: g(s) \neq s\} \text {. }
$$

If there exist $x, y \in S$ such that $\operatorname{supp}(g) \subseteq[x, y]$, then $g$ is said to have bounded support. If $G$ is $o-2$ transitive on $\langle S, \leqq\rangle$ and has no element of bounded support other than the identity, $G$ is called pathological.

If $G$ is $o-2$ transitive on $\langle\mathrm{S}, \leqq\rangle$, then the set of elements of $G$ of bounded support is an $l$-ideal of $G$. Consequently,

Theorem (Holland (5)). If $G$ is $0-2$ transitive on $\langle S, \leqq\rangle$, then $G$ is l-simple if all its elements are of bounded support. If, in addition, $G$ contains an elementother than the identity-of bounded support, the converse is true.

We must now examine pathological $o-2$ transitive $l$-groups. Examples of such groups are sparse in the literature; essentially, the only ones known can be found in (4) and (8) and they are $l$-simple. This led to the conjecture that every pathological $0-2$ transitive $l$-group is $l$-simple (see (6) and (8)). Were it true, it would yield a complete classification of those $l$-simple $l$-groups which have a transitive 0 -primitive representation. Actually, it is false. To prove this, we will provide new pathological $0-2$ transitive $l$-groups and, in particular, will prove a theorem concerning free $l$-groups (free in the category of $l$-groups) on an infinite set of generators.

For a further discussion of ordered permutation groups, (6) is an excellent expository article.

\section{Ultraproducts of pathological $o$-2 transitive $l$-groups}

Suppose that for each $i \in I, G_{i}$ is a pathological $o-2$ transitive $l$-subgroup of Aut $\left(\left\langle S_{i}, \leqq_{i}\right\rangle\right)$. Let $D$ be an ultrafilter on $I$. Then $G=\Pi_{D} G_{i}$ is a pathological $o-2$ transitive $l$-subgroup of Aut $(\langle S, \leqq\rangle)$ where $S=\Pi_{D} S_{i}$ and $s_{D} \leqq t_{D}$ if and only if $\left\{i \in I: s(i) \leqq_{i} t(i)\right\} \in D$ (see (1) and (2) for further background on ultraproducts). This fact is easily verified. 
Let $I=\omega$, the first infinite ordinal and let $D$ be any non-principal ultrafilter on $I$. For each $i \in I$, let $G_{i}=H=\left\{h \in\right.$ Aut $(\langle\mathbf{R}, \leqq\rangle): h f_{0}^{n}=f_{0}^{n} h$ for some positive integer $n\}$ where $f_{0} \in$ Aut $(\langle\mathbf{R}, \leqq\rangle)$ is defined by: $f_{0}(r)=r+1$ for all $r \in \mathbf{R}$. Then $H$ is an $l$-simple pathological $o$-transitive $l$-group (4). Observe that $f_{0}^{n+1} h^{-1} f_{0}^{n} h$ for any $h \in H$ and any positive integer $n$ (for if $n$ is a positive integer and $h \in H$ are such that $f_{0}^{n+1} \leqq h^{-1} f_{0}^{n} h$, then for all

$r \in \mathbf{R}$,

$$
f_{0}^{n+1}\left(h^{-1}(r)\right) \leqq h^{-1} f_{0}^{n} h\left(h^{-1}(r)\right)=h^{-1}(r+n) ;
$$

i.e.

$$
h^{-1}(r)+n+1 \leqq h^{-1}(r+n) \text {. }
$$

There exists a positive integer $m$ such that $f_{0}^{m} h=h f_{0}^{m}$. Replacing $r$ by $0, n$, $2 n, \ldots, m n$ in $\left({ }^{*}\right)$ we obtain

$$
h^{-1}(0)+m(n+1) \leqq h^{-1}(m n)=h^{-1}(0+m n)=h^{-1}(0)+m n,
$$

Aut $\left(\left\langle\Pi_{D} \mathbf{R}, \leqq\right\rangle\right)$ which is not $l$-simple since the $l$-ideal generated by a contradiction). Then $G=\Pi_{D} G_{i}$ is a pathological $o$-2 transitive $l$-subgroup of

$$
\left\{k_{D}: k(i)=k(j) \text { for all } i, j \in I\right\}
$$

is proper (cf. the result for groups, see (2)). It can also be shown that $G$ is not "periodic" in any sense even though each $H_{i}$ is (this is a more general notion of periodic than that given in the introduction). This example indicates that the conjectures of (8) are false. Moreover, " $l$-simple" cannot be expressed in first-order logic (for if a first order sentence is true in $\mathfrak{A}_{i}$ for each $i \in I$, then it is true in $\Pi_{D} \mathfrak{A}_{i}$ for any ultrafilter $D$ on $I$ ).

\section{Free $l$-groups}

Let $\kappa$ be an infinite cardinal. $F_{\kappa}$ will denote the free $l$-group generated by $\left\{x_{\alpha}: \alpha<\kappa\right\}$ where $x_{\alpha} \neq x_{\beta}$ if $\alpha, \beta<\kappa$ and $\alpha \neq \beta$. Let $\mathbf{Z}$ be the set of integers and $\langle\mathbf{Q}, \leqq\rangle$ the set of rationals under the usual ordering.

Lemma. $F_{w}$ is l-isomorphic to an l-subgroup of Aut $(\langle\mathbf{Q}, \leqq\rangle)$ which has no element (other than the identity) of bounded support.

Proof. Let $\left\{I_{n}: n \in \mathbf{Z}\right\}$ be any set of bounded non-empty open intervals in $\left\langle\mathbf{Q} \leqq>\right.$ such that if $m, n \in \mathbf{Z}$ and $m<n$, then $I_{m}<I_{n}$ (if $q \in I_{m}$ and $r \in I_{n}$, then $q<r)$ and if $q \in \mathbf{Q}$, there exists $p \in \mathbf{Z}$ such that $p>0$ and $I_{-p}<q<I_{p}$. Since the first order theory of dense total ordering without endpoints is $\omega$-categorical, see (1), $\left\langle I_{n}, \leqq\right\rangle$ is isomorphic to $\langle\mathbf{Q}, \leqq\rangle$ for each $n \in \mathbf{Z}$. The proof of Theorem 2 of (3) shows that $F_{\omega}$ can be $l$-embedded in $\operatorname{Aut}(\langle\mathbf{Q}, \leqq\rangle)$ and so in

$$
\text { Aut }\left(\left\langle I_{n}, \leqq\right\rangle\right)
$$

for each $n \in \mathbf{Z}$. Let $\bar{x}_{m, n}$ be the image of $x_{m}$ in Aut $\left(\left\langle I_{n}, \leqq\right\rangle\right)$ for $m, n \in \mathbf{Z}$ and $m \geqq 0$. Define $f_{m} \in$ Aut $(\langle\mathbf{Q}, \leqq\rangle)$ as follows: Let $q \in \mathbf{Q}$; if $q \in I_{n}$ for some $n \in \mathbf{Z}$, let $f_{m}(q)=\bar{x}_{m, n}(q)$ and if $q \in \bigcup\left\{I_{n}: n \in \mathbf{Z}\right\}$, let $f_{m}(q)=q$. Let $W$ be the subgroup of Aut $(\langle\mathbf{Q}, \leqq\rangle)$ generated by $\left\{f_{m}: m \in \omega\right\}$. The map $\phi: F_{\boldsymbol{\omega}} \rightarrow W$ 
defined by $\phi\left(x_{m}\right)=f_{m}$ is an $l$-isomorphism of $F_{\omega}$ on to $W$. Indeed, by construction, the only element of $W$ of bounded support is the identity so the lemma is proved.

Theorem. $F_{\omega}$ is l-isomorphic to a pathological o-2 transitive l-subgroup of Aut $(\langle\mathbf{Q}, \leqq\rangle)$.

Proof. Let $A=\{(q, r): q, r \in \mathbf{Q}$ and $q<r\}$ and $B=A \times A$. The cardinality of $B$ is $\omega$ and so there exists a one-to-one function $\psi$ of $\omega$ onto $B$. Denote $\psi(m)$ by $\left(a_{m}, b_{m}, c_{m}, d_{m}\right)$. Define a sequence of bounded open intervals $X_{m}$ in $\langle\mathbf{Q}, \leqq\rangle$ such that $X_{0} \subseteq X_{1} \subseteq \ldots \subseteq X_{m} \subseteq \ldots$ and $\left[a_{m}, b_{m}\right],\left[c_{m}, d_{m}\right] \subseteq X_{m}$ for each $m \in \omega$. For each $m \in \omega,\left\langle X_{m}, \leqq\right\rangle$ is isomorphic to $\langle\mathbf{Q}, \leqq\rangle$ and so there exists $k_{m} \in$ Aut $\left(\left\langle X_{m}, \leqq\right\rangle\right)$ such that $k_{m}\left(a_{m}\right)=c_{m}$ and $k_{m}\left(b_{m}\right)=d_{m}$. Let $\left\{I_{n}: n \in \mathbf{Z}\right\}$ be as in the the proof of the lemma subject to the extra condition that

$$
I_{-(m+1)}<X_{m}<I_{m+1}
$$

for each $m \in \omega$. Let $\bar{x}_{m n}$ be defined as in the proof of the lemma and define $f_{m} \in$ Aut $(\langle\mathbf{Q}, \leqq\rangle)$ for $m \in \omega$ as follows: let $q \in \mathbf{Q}$; if $q \in X_{m}$, let $f_{m}(q)=k_{m}(q)$; if $q \notin I_{p}$ for some $p \in \mathbf{Z}$ such that $|p|>m$, let $f_{m}(q)=\bar{x}_{m, n}(q)$; if $q \notin X_{m} \bigcup\left\{I_{p}\right.$ : $|p|>m\}$, let $f_{m}(q)=q$. Let $W$ be the $l$-subgroup of Aut $(\langle\mathbf{Q}, \leqq\rangle)$ generated by $\left\{f_{m}: m \in \omega\right\}$. By the coding of $B, W$ is an $o-2$ transitive $l$-subgroup of Aut $(\langle\mathbf{Q}, \leqq\rangle)$ which is pathological by the construction. The map $\phi: F_{\omega} \rightarrow W$ defined by $\phi\left(x_{m}\right)=f_{m}$ is an $l$-isomorphism of $F_{\omega}$ on to $W$.

Corollary 1. There exist pathological o-2 transitive l-groups which are neither l-simple nor periodic in any sense.

Note that the proof of the lemma would apply to any free $l$-group on a finite number of generators in place of $F_{w}$. If $\kappa$ is an infinite cardinal such that whenever there exists an order-isomorphism between two subsets of $S$ of cardinality less than $\kappa$, the order-isomorphism can be extended to some element of $G$, then $G$ is said to be $o-\kappa$ transitive on $\langle S, \leqq\rangle$. Any $l$-group which is $o-2$ transitive on $\langle S, \leqq\rangle$ is $o-\omega$ transitive on $\langle S, \leqq\rangle$. However, the ideas of the proof of the theorem could have been used to show directly that $F_{\omega}$ is $l$-isomorphic to a pathological $o-\omega$ transitive $l$-subgroup of Aut $(\langle\mathbf{Q}, \leqq\rangle)$.

Let $\alpha$ be an ordinal. $\langle S, \leqq\rangle$ is said to be an $\alpha$-set if and only if $S$ has cardinality $\aleph_{\alpha}$ and whenever $X, Y \subseteq S$ are such that $|X \cup Y|<\aleph_{\alpha}$ and $X<Y$, there exists $s \in S$ such that $X<\{s\}<Y .\langle\mathbf{Q}, \leqq\rangle$ is a 0 -set. For any $\alpha$, there exists at most one $\alpha$-set (to within isomorphism). Moreover, the existence of $\alpha$-sets for all $\alpha$ such that $\aleph_{\alpha}$ is regular is equivalent to the generalised continuum hypothesis (G.C.H.). It was essentially shown in (9) that if $\langle S, \leqq\rangle$ is an $\alpha$-set, it is $o-\aleph_{\alpha}$ transitive; alternatively, $\langle S, \leqslant\rangle$ is saturated and so homogeneousthat is, $o-\aleph_{\alpha}$ transitive-(see (1)). Therefore the proof of the theorem yields:

Corollary 2 (G.C.H.). For any ordinal $\alpha, F_{\omega_{\alpha}}$ is l-isomorphic to a pathological $o-\aleph_{\alpha}$ transitive l-subgroup of Aut $\left(\left\langle S_{\alpha}, \leqq\right\rangle\right)$ where $\left\langle S_{\alpha}, \leqq\right\rangle$ is an $\alpha$-set. 
The following result can be found in (5):

Let $G$ be a transitive $l$-subgroup of Aut $(\langle S, \leqq\rangle$ ) and $e<g \in G$ ( $e$ is the identity function). $g$ has bounded support if and only if the sentence

$$
\exists h \forall k\left(k \geqq e \Rightarrow g \wedge k^{-1} h^{-1} g h k=e\right)
$$

holds in $G$. If $G$ has no positive element (other than the identity) of bounded support, then $G$ has no element (other than the identity) of bounded support. Consequently, the $o$ - 2 transitive $l$-group $G$ is pathological if and only if the sentence

$$
\forall g\left(g \ngtr e \vee \forall h \exists k\left(k \geqq e \& g \wedge k^{-1} h^{-1} g h k \neq e\right)\right)
$$

holds in $G$. This is a $\Pi_{2}^{0}$ sentence and so is preserved under 1-sandwiches (see (1)). It is not preserved under $l$-homomorphic images since it is satisfied in all free $l$-groups (by the Lemma and Corollary 2 ), every $l$-group is an $l$-homomorphic image of a free $l$-group and there exist non-pathological $o-2$ transitive l-groups. Hence (2) is not equivalent to a positive sentence (see (1)).

In (3, Theorem 3$)$ it was shown that an $l$-group $G$ is $l$-isomorphic to a transitive $l$-subgroup of some Aut $(\langle S, \leqq\rangle)$ if and only if there exists a prime convex $l$-subgroup $C$ of $G$ such that $C$ contains no $l$-ideal of $G$ other than $\{e\}$. ( $C$ is prime if $f, g \in G$ and $f \wedge g=e$ imply $f \in C$ or $g \in C$.) Such a subgroup $C$ is called a representing subgroup of $G . \quad G$ is $l$-isomorphic to an $o-2$ transitive $l$-subgroup of some Aut $(\langle S, \leqq\rangle)$ if and only if there exists a representing subgroup $C$ of $G$ such that if $e \leqq f_{i} \in G \backslash C(\mathrm{i}=1,2)$, there exists $g \in C$ such that $C f_{1} g=C f_{2}$. Such a representing subgroup will be called a strong representing subgroup. Thus:

Corollary 3. Let $F$ be a free l-subgroup and $K$ an l-ideal of $F . \quad F / K$ is l-isomorphic to a pathological o-2 transitive l-subgroup of some Aut $(\langle S, \leqq\rangle)$ if and only if there exists a strong representing subgroup $C$ of $F$ such that $C \supseteq K, C$ contains no l-ideal of $F$ which properly contains $K$ and the sentence (2) holds in $F / K$.

This yields an algebraic method of determining, inside free $l$-groups, which quotients have pathological $o$ - 2 transitive $l$-isomorphic images. Unfortunately, this result is very limited since it leaves unanswered many natural questions; e.g. do there exist pathological $o-2$ transitive $l$-groups all of whose elements (other than the identity) have a finite (bounded) set of fixed points? However, a thorough examination of pathological $o-2$ transitive $l$-groups seems necessary so as to make possible a characterisation of the $l$-simple $l$-groups which have an $o$-primitive transitive representation.

\section{REFERENCES}

(1) C. C. Chang and H. J. Keisler, Model Theory (North Holland, 1973).

(2) T. E. Frayne, A. C. Morel and D. S. Scott, Reduced direct products, Fund. Math. 51 (1962), 195-228.

E.M.S. $-19 / 2-K$ 
(3) W. C. Holland, The lattice-ordered groups of automorphisms of an ordered set, Michigan Math. J. 10 (1963), 399-408.

(4) W. C. Holland, Transitive lattice-ordered permutation groups, Math. $Z$. 87 (1965), 420-433.

(5) W. C. Holland, A class of simple lattice-ordered groups, Proc. Amer. Math. Soc. 16 (1965), 326-329.

(6) W. C. Holland, Ordered permutation groups, Permutations (actes du colloque Paris, juillet 1972), (Gauthier-Villars, 1974).

(7) S. H. MCCleary, o-primitive ordered permutation groups, Pacific J. Math. 40 (1972), 349-372.

(8) S. H. MCClEARY, o-2 transitive ordered permutation groups, Pacific J. Math. 49 (1973), 425-430.

(9) S. H. MCClearY, The lattice-ordered group of automorphisms of an $\alpha$-set Pacific J. Math. 49 (1973), 417-424.

Bowling Green State University

Bowling Green, OHIO 43403

U.S.A. 